References

1. Almeida PP, Novaes MA, Bressan RA, Lacerda AL. Review: executive functioning and cannabis use. Rev Bras Psiquiatr. 2008;30(1):69-76.

2. Aharonovich E, Brooks AC, Nunes EV, Hasin DS. Cognitive deficits in marijuana users: effects on motivational enhancement therapy plus cognitive behavioral therapy treatment outcome. Drug Alcohol Depend. 2008;95(3):279-83.

3. Solowij N, Pesa N. Cognitive abnormalities and cannabis use. Rev Bras Psiquiatr. 2010;32(Suppl 1):S31-40.

\section{Depressão durante a gravidez no sistema público de saúde brasileiro}

\section{Depression during pregnancy in the Brazilian public health care system}

Caros Editores,

Gostaríamos de parabenizar os autores Silva et al. pelo trabalho recém-publicado na Revista Brasileira de Psiquiatria ${ }^{1}$. O artigo avalia a prevalência e fatores associados à depressão em gestantes atendidas por meio do sistema público de saúde da cidade de Pelotas. Trata-se de pesquisa de enorme relevância para a saúde pública que aborda tema frequentemente relegado a plano secundário na assistência às gestantes. O estudo mostra que $21 \%$ das participantes apresentam episódio depressivo na gravidez, ressaltando a importância do papel do profissional de saúde no diagnóstico precoce e adoção de medidas terapêuticas. No entanto, gostaríamos de fazer um reparo ao trabalho e comentar seus resultados.

$\mathrm{Na}$ introdução, os autores afirmam que este é primeiro estudo nacional em amostra representativa da população. Nosso artigo "Common mental disorders during pregnancy: prevalence and associated factors among low-income women in São Paulo, Brazil”, publicado no periódico Archives of Womens' Mental Health, em
4. Pope HG, Gruber AJ, Hudson JI, Huestis MA, Yurgelun-Todd D. Neuropsychological performance in long-term cannabis users. Arch Gen Psychiatry. 2001;58(10):909-15.

5. Bolla KI, Brown K, Eldreth D, Tate K, Cadet JL. Dose-related neurocognitive effects of marijuana use. Neurology. 2002;59(9):1337-43.

6. Cunha PJ, Nicastri S, Andrade AG, Bolla KI. The Frontal Assessment Battery (FAB) reveals neurocognitive dysfunction in substancedependent individuals in distinct executive domains: abstract reasoning, motor programming, and cognitive flexibility. Addict Behav. 2010;35(10):875-81.

$2009^{2}$, avaliou um grupo de 831 gestantes com características sociodemográficas muito semelhantes atendidas em pré-natal nas unidades de atenção primária do sistema público de saúde da região Oeste da cidade de São Paulo. Gostaríamos de destacar que a prevalência de depressão/ansiedade avaliada por meio da Clinical Interview Schedule-Revised (CIS-R) foi igualmente de 20\%. Quanto aos fatores associados à depressão, também observamos que história de tratamento psiquiátrico prévio, uso de álcool e tabagismo mostraram-se fortemente associados com prevalência de depressão. Também do mesmo modo que o observado por Silva et al. ${ }^{1}$, não ter parceiro $(\mathrm{p}=0,09)$ e baixa escolaridade $(0,02)$ se associaram com depressão apenas na análise univariada. Por outro lado, diferentemente dos resultados do estudo de Pelotas, não encontramos associação entre depressão na gestação e número de gestações anteriores, gravidez planejada ou ter mais idade. Pelo contrário, em nossa amostra, a idade mais jovem na gestação atual é que apresentou associação estatisticamente significante com o desfecho em questão, sugerindo que talvez fatores contextuais possam modificar a associação entre idade e depressão na gravidez.

Por se tratar de problema tão prevalente e com potenciais consequências para a mulher e para o bebê, a exemplo de nossos colegas de Pelotas, acreditamos que novas pesquisas nacionais sobre depressão no ciclo gravídico-puerperal são necessárias para auxiliar no desenvolvimento de programas e políticas públicas que permitam melhorar efetivamente a assistência às gestantes oferecida pelo Sistema Único de Saúde do país.

Alexandre Faisal-Cury, Paulo Menezes Departamento de Medicina Preventiva, Faculdade de Medicina, Universidade de São Paulo (USP), São Paulo, SP, Brasil

\title{
Disclosures
}

\begin{tabular}{|c|c|c|c|c|c|c|c|}
\hline $\begin{array}{l}\text { Writing group } \\
\text { member }\end{array}$ & Employment & $\begin{array}{l}\text { Research } \\
\text { grant }^{1}\end{array}$ & $\begin{array}{c}\text { Other research grant } \\
\text { or medical continuous } \\
\text { education }\end{array}$ & $\begin{array}{l}\text { Speaker's } \\
\text { honoraria }\end{array}$ & $\begin{array}{c}\text { Ownership } \\
\text { interest }\end{array}$ & $\begin{array}{l}\text { Consultant/ } \\
\text { Advisory } \\
\text { board }\end{array}$ & Other ${ }^{3}$ \\
\hline Alexandre Faisal-Cury & USP & - & - & - & - & - & - \\
\hline Paulo Menezes & USP & - & - & - & - & - & - \\
\hline
\end{tabular}


Referências

1. Silva RA, Jansen K, Souza LD, Moraes IG, Tomasi E, Silva Gdel G, Dias Mde $S$, Pinheiro RT. Depression during pregnancy in the Brazilian public health care system. Rev Bras Psiq. 2010;32(2):139-44.

2. Faisal-Cury A, Menezes P, Araya R, Zugaib M. Common mental disorders during pregnancy: prevalence and associated factors among low-income women in São Paulo, Brazil. Arch Womens Ment Health. 2009;12(5):335-43.

\section{Resposta à carta de Faisal-Cury et al. "Depressão durante a gravidez no sistema público de saúde brasileiro"}

\section{Reply to Faisal-Cury et al.'s letter "Depression during pregnancy in the Brazilian public health care system"}

Caros Editores,

É uma satisfação para a equipe de pesquisa do Programa de PósGraduação em Saúde e Comportamento da Universidade Católica de Pelotas receber a carta aos editores intitulada "Depressão durante a

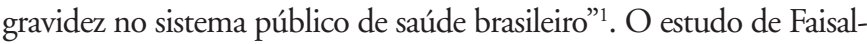

Cury é um ótimo trabalho com a mesma população-alvo e foi citado por nosso grupo no artigo intitulado "Transtornos mentais comuns (TMC), autoestima e fatores associados em gestantes'3 ${ }^{3}$ já aprovado no periódico Cadernos de Saúde Pública e que aguarda publicação. Entretanto, não foi incluído em nossa revisão, nem o consideramos um estudo antecedente ao nosso presente artigo, uma vez que seu estudo apresenta uma diferente medida de avaliação e desfecho. $\mathrm{O}$ estudo de Faisal-Cury utilizou uma entrevista clínica que tem por objetivo avaliar sintomas psiquiátricos em seu conjunto (transtornos mentais), que incluem inquietação, irritabilidade, ansiedade, preocupação somática, problemas do sono e da concentração, fadiga, humor depressivo, comportamento compulsivo e obsessivo, hipocondria, fobias e pânico, agrupados em TMC. A investigação verificou os fatores associados a este conjunto, enquanto nosso estudo verificou fatores associados especificamente o quadro de depressão gestacional, por meio da Edinburgh Postnatal Depression Scale (EPDS), instrumento com validação para esta população. Mesmo com tais diferenças metodológicas, os manuscritos reforçam a necessidade de que se atente para a necessidade de avaliação e tratamento de transtornos mentais na gestação e puerpério.

Ricardo Azevedo da Silva, Karen Jansen, Luciano Dias Mattos de Souza, Inácia Gomes da Silva Moraes, Elaine Tomasi, Giovanna Del Grande da Silva, Michelle Souza Dias, Ricardo Tavares Pinheiro Universidade Católica de Pelotas, Pelotas, RS, Brasil

Financiamento e conflito de interesses

\begin{tabular}{|c|c|c|c|c|c|c|c|}
\hline $\begin{array}{l}\text { Membro do grupo } \\
\text { de autores }\end{array}$ & $\begin{array}{l}\text { Local de } \\
\text { trabalho }\end{array}$ & $\begin{array}{l}\text { Verba de } \\
\text { pesquisa }\end{array}$ & $\begin{array}{c}\text { Outro apoio à } \\
\text { pesquisa ou educação } \\
\text { médica continuada }\end{array}$ & $\begin{array}{l}\text { Honorários } \\
\text { de } \\
\text { palestrantes }\end{array}$ & $\begin{array}{l}\text { Participação } \\
\text { acionária }\end{array}$ & $\begin{array}{l}\text { Consultorl } \\
\text { conselho } \\
\text { consultivo }\end{array}$ & Outro $^{3}$ \\
\hline $\begin{array}{l}\text { Ricardo Azevedo da } \\
\text { Silva }\end{array}$ & UCPel & - & - & - & - & - & - \\
\hline Karen Jansen & UCPel & - & - & - & - & - & - \\
\hline $\begin{array}{l}\text { Luciano Dias Mattos } \\
\text { de Souza }\end{array}$ & UCPel & - & - & - & - & - & - \\
\hline $\begin{array}{l}\text { Inácia Gomes da } \\
\text { Silva Moraes }\end{array}$ & UCPel & - & - & - & - & - & - \\
\hline Elaine Tomasi & UCPel & - & - & - & - & - & - \\
\hline $\begin{array}{l}\text { Giovanna Del Grande } \\
\text { da Silva }\end{array}$ & UCPel & - & - & - & - & - & - \\
\hline Michelle Souza Dias & UCPel & - & - & - & - & - & - \\
\hline $\begin{array}{l}\text { Ricardo Tavares } \\
\text { Pinheiro }\end{array}$ & UCPel & - & - & - & - & - & - \\
\hline
\end{tabular}

* Modesto

** Significativa

*** Significativa. Montantes fornecidos à instituição do autor ou a colega onde o autor tem participação, não diretamente ao autor. Nota: UCPel = Universidade Católica de Pelotas.

Mais informações, consultar as Instruções aos Autores.

Referências

1. Silva RA, Jansen K, Souza LDM, Moraes IGS, Tomasi E, Silva GDG, Dias MS, Pinheiro RT. Depression during pregnancy in the Brazilian public health care system. Rev Bras Psiquiatr. 2010;32(2):139-44.

2. Faisal-Cury A, Menezes P, Araya R, Zugaib M. Common mental disorders during pregnancy: prevalence and associated factors among low- income women in São Paulo, Brazil. Arch Womens Ment Health. 2009; $12: 335-43$.

3. Silva RA, Ores LC, Mondin TC, Rizzo RN, Moraes IGS, Jansen K, Pinheiro RT. Transtornos mentais comuns e auto-estima na gestação: prevalência e fatores associados. Cad Saude Publica. In press, 2010. 